\title{
Aspectos epidemiológicos de Cryptosporidium spp. en terneros del estado Táchira en Venezuela ${ }^{1}$
}

\author{
Epidemiological aspects of Cryptosporidium spp. in calves of Tachira State in \\ Venezuela
}

Carlos D. Moreno-Manresa ${ }^{2}$, Angela Arellano-Semidey ${ }^{3}$, Cindy Martin-Pérez ${ }^{4}$

\begin{abstract}
Resumen
Los procesos diarreicos son los más comúnmente involucrados en las pérdidas por muertes o disminución de crecimiento, y se dan en etapas tempranas de la vida de los animales de producción. En Venezuela, particularmente en el estado Táchira, se ha determinado en terneros la presencia de protozoarios del género Cryptosporidium, pero no se ha evaluado suficientemente su relación con manifestaciones clínicas de enfermedad gastrointestinal. El objetivo de este trabajo fue evaluar la epidemiología de Cryptosporidium spp. en los terneros de rebaños del estado Táchira y su relación con manifestaciones clínicas de enfermedad digestiva. Se muestrearon 338 terneros aleatoriamente durante el año 2008. Las variables epidemiológicas estudiadas fueron: a) la edad en meses, tomada de los registros existentes en las unidades de producción; b) el sexo del animal; c) la zona del estado Táchira (norte o sur); d) presencia de signos evidentes de diarrea; y e) positividad o negatividad a criptosporidios al examen coprológico. Del total, un $27 \%$ mostró positividad a Cryptosporidium spp. por la técnica de Zielh Neelsen modificado. El estudio sugiere que este género de protozoarios constituye un factor de riesgo para la presentación de diarreas en terneros desde el nacimiento al destete en el estado Táchira (OR $>1 ; \mathrm{p}<0,05)$; donde el 18,8\% de los casos de diarreas indiferenciadas pueden guardar relación con la presencia del parásito $(\mathrm{p}<0,05)$. Las infecciones resultaron menos comunes en los terneros mayores $(\mathrm{OR}<1$; $\mathrm{p}<0,01)$, sin embargo, la edad no actúa como un factor determinante para la presentación de diarrea en animales ya infectados. El sexo no se presentó como factor de riesgo para la infección por Cryptosporidium spp.
\end{abstract}

Palabras clave: bovinos, epidemiología, criptosporidiosis, infecciones por protozoos.

\begin{abstract}
Diarrheic processes are most commonly involved in losses due to mortality or decline of growth and occur at early ages in the farm animals. In Venezuela, particularly in the Tachira state, the presence of protozoa of the genus Cryptosporidium has been determined in calves, but its relationship with a clinical gastrointestinal disease is not sufficiently evaluated. The objective of this work was to evaluate the epidemiology of Cryptosporidium spp. in the calves of herds in Tachira state and its relation with clinical manifestations of a digestive disease. 338 calves were
\end{abstract}

\footnotetext{
Recibido: 23 de noviembre, 2017. Aceptado: 19 de marzo, 2018. Este trabajo formó parte de un conjunto de muestreos de campo realizados por el Laboratorio de Investigación en Fisiología y Sanidad Animal (LIFSA), Venezuela.

2 Universidad Nacional Experimental del Táchira, Laboratorio de Investigación en Fisiología y Sanidad Animal. Paramillo, San Cristóbal. 5001. Venezuela.cdmoreno@unet.edu.ve (autor para correspondencia).

3 Boeringer Ingelheim, CA. Chuao. Caracas 1061 Venezuela. aias231980@gmail.com

4 Hospital for Children Headquarters. Tampa, Florida. 33607. USA. cindyperez31@gmail.com
} 
randomly sampled during 2008. The epidemiological variables studied were: a) the age in months, taken from existing records in production units; b) the gender of the animal; c) the Tachira state zone (north or south); d) presence of obvious signs of diarrhea; and e) positivity or negativity to cryptosporidium upon coprological examination. $27 \%$ of the samples showed positivity to Cryptosporidium spp. by the Zielh Neelsen modified technique. The study suggests that this genus of protozoa constitutes an important risk factor for the presentation of diarrhea in calves from birth to weaning in the Tachira state $(\mathrm{OR}>1, \mathrm{p}<0.05)$; where $18.8 \%$ of cases of undifferentiated diarrhea may be related to the presence of the parasite $(\mathrm{p}<0.05)$. Infections were less common in older calves $(\mathrm{OR}<1 ; \mathrm{p}<0.01)$; however, age did not act as a determinant factor for the presentation of diarrhea in already infected animals. Gender does not present as a risk factor for infection by Cryptosporidium spp.

Keywords: livestock, epidemiology, cryptosporidiosis, protozoal infections.

\section{Introducción}

Los procesos diarreicos en animales de producción son los más comúnmente involucrados en las pérdidas por muertes o disminución del crecimiento, y se dan en etapas tempranas de la vida. En bovinos jóvenes, los agentes etiológicos más comunes son: rotavirus bovino (BRV), coronavirus bovino (BCoV), virus de la diarrea viral bovina (BVDV), Salmonella enterica, Escherichia coli, Clostridium perfringens y Cryptosporidium parvum, así como nuevos patógenos emergentes: torovirus bovino (BToV), calicivirus, norovirus bovino (BNoV) y nebovirus (Cho y Yoon, 2014). Se ha reportado la frecuente asociación de Cryptosporidium spp. con otros enteropatógenos en bovinos que, en áreas periurbanas constituyen focos de contaminación para los seres humanos (Brar et al., 2017), siendo una infección muy frecuente en países como: Estados Unidos, Canadá, Reino Unido, Irlanda, Suecia, Alemania, Bélgica, Holanda, Italia, España, Portugal, Hungría, Serbia y Montenegro, Eslovenia, Japón, India, Australia y Nueva Zelandia, en donde se han observado prevalencias importantes en terneros de muy corta edad (Wang et al., 2011).

En Venezuela, particularmente en el estado Táchira, se ha determinado la presencia de protozoarios del género Cryptosporidium en terneros (González y Moreno, 2003). El género Cryptosporidium pertenece al Phylum Apicomplexa, clase Coccidia, orden Eucoccidiorida, familia Cryptosporidiidae (Fayer, 2010). A pesar de las similitudes en ciclos de vida, varias características distinguen al género Cryptosporidium del resto de los coccidios. Dentro de estas se encuentra: amplitud de especies hospedadoras con diferentes sensibilidades, capacidad de autoinfección por la rápida esporulación dentro del hospedador, esporas de muy reducido tamaño, localización intracelular y extracitoplasmática en la célula hospedadora, y una terapéutica con escasos principios activos a los que el Cryptosporidium es sensible.

Se han reconocido veintiséis especies del género Cryptosporidium con base morfológica, biológica y molecular; de las cuales veinte especies y genotipos han sido reportados en humanos (Ryan et al., 2014). $C$. parvum es considerada como la principal especie en rumiantes (Andrade et al., 1986; Surumay y Alfaro, 2000), de la cual se han identificado seis subespecies potencialmente zoonóticas (Mariné-Oliveira et al., 2016). Debido a su importancia como parásito, esencialmente digestivo (Bhatia, 2000; Aldeyarbi et al., 2016), se realizó este trabajo teniendo como objetivo evaluar la epidemiología de Cryptosporidium spp. en los terneros de rebaños del estado Táchira y su relación con manifestaciones clínicas de enfermedad digestiva. 


\section{Materiales y métodos}

La investigación se realizó durante el año 2008 en el Laboratorio de Investigación de Fisiología y Sanidad Animal (LIFSA), unidad de investigación adscrita a la Universidad Nacional Experimental del Táchira (UNET). Consistió en un estudio observacional transversal epidemiológico para Cryptosporidium spp. en terneros. Las variables epidemiológicas estudiadas fueron: $a$ ) la edad en meses, tomada de los registros existentes en las unidades de producción; $b$ ) el sexo del animal; $c$ ) la zona del estado Táchira (norte o sur); $d$ ) presencia de signos evidentes de diarrea; y e) positividad o negatividad a criptosporidios al examen coprológico. El estudio incluyó los municipios más destacados en la explotación bovina del estado Táchira. La zona norte del estado Táchira en Venezuela es zona de influencia estacional de la cuenca del Lago de Maracaibo, y en esta fueron tomados en cuenta los municipios de importancia ganadera: García de Hevia, Panamericano y Samuel Darío Maldonado. En la zona sur del estado Táchira, separada geográficamente de la anterior por la cadena montañosa de la Cordillera de los Andes, es zona de influencia de la región llanera, en esta se seleccionaron los municipios: Fernández Feo, Libertador y Uribante (Castellanos et al., 2013). Ambas zonas se caracterizan por tener bosque húmedo tropical de suelos aluviales mixtos de pie de monte andino (Leal et al., 1999), con climas muy cálidos y muy lluviosos, con una estacionalidad más marcada en la zona sur, dada su influencia del clima propio de los llanos venezolanos (Silva-León, 2010).

En la zona norte, las fincas se manejan en los grupos de tecnología básica y media casi en su totalidad (96\%), en la zona sur, hay mayor distribución en los grupos tecnológicos, con predominio (68\%) de grupos de tecnología básica y alta (Castellanos et al., 2013). El tipo racial de bovino predominante en estas zonas corresponde a cruzamientos entre razas de Bos indicus y Bos taurus siguiendo muchas veces las estrategias de criadores y agrotécnicos para mantener un tipo doble propósito (carne-leche) (Aranguren-Méndez et al., 2007).

Para una población total reportada de 104606 bovinos lactantes (SASA, 2005), se calculó el tamaño de la muestra mediante el software WinEpi (Universidad de Zaragoza). Se determinó un tamaño mínimo de 265 terneros tomados al azar para estimar la prevalencia de Cryptosporidium spp. en bovinos lactantes del estado Táchira. Se utilizó como prevalencia esperada $22,1 \%$ y un error esperado de 5\%, con un nivel de confianza de $95 \%$, válido para poblaciones de más de 1000 individuos (Surumay y Alfaro, 2000).

Los datos fueron agrupados por edades desde los uno a los siete meses, se tomó en cuenta en cada grupo, cuántos individuos presentaron signos de diarrea.

Las unidades de producción muestreadas fueron de doble propósito. Los animales fueron seleccionados al azar entre los terneros presentes en cada finca, independientemente de la presencia o no de diarrea.

Se realizó un examen clínico general de cada animal muestreado, con especial énfasis en la presencia o ausencia de signos de enfermedad digestiva.

El método de diagnóstico utilizado en la determinación de la presencia del parásito, fue la técnica de coloración de Ziehl Neelsen modificado para criptosporidios (Angus, 1987; Contreras, 2000; Rekha et al., 2016) por observación a microscopio óptico binocular. Los cálculos de prevalencia se realizaron mediante las aplicaciones Win Episcope y Win Epi Tasas del software epidemiológico WinEpi (Facultad de Veterinaria, Universidad de Zaragoza, España); los cálculos de odds ratio y regresión logística fueron obtenidos con el software Epi info ${ }^{\mathrm{TM}}$ (CDC, Center for Disease Control and Prevention).

Se analizó el factor exposición al parásito, expresado como animales con resultado coprológico positivo a criptosporidios, relacionándolo con la presencia o no de signos clínicos de diarrea en los terneros muestreados. Se determinó el odds ratio (OR), entendido como el exceso o defecto de ventaja ( odds») que tienen los individuos expuestos de presentar la enfermedad o condición (diarrea), frente a no padecerla, respecto a la ventaja de los individuos no expuestos de presentar diarrea frente a la posibilidad de no presentarla. Simultáneamente, se determinó la razón de prevalencia (RP), que se define en términos de cuántas veces es más probable que los individuos expuestos presenten la enfermedad o condición respecto a aquellos individuos no expuestos (Schiaffino et al., 2003) con sus 
respectivas comprobaciones estadísticas (aproximación logarítmica y de $\chi^{2}$.) Se calculó además, el riesgo atribuible (RA), que representa la expresión de la relación de la exposición con la respuesta (Orellana et al., 2013); la fracción etiológica (FE), que es una relación causal en la que el factor de exposición participe como responsable de estas manifestaciones clínicas; y la fracción atribuible de la población (FAP), la cual se consideró como el porcentaje de la población total de terneros que pueden estar manifestando signos clínicos relacionados con la presencia del parásito.

\section{Resultados}

\section{Positividad del parasitismo por Cryptosporidium spp. en terneros del estado Táchira}

La tasa de infección estimada de Cryptosporidium spp. en terneros (calculada con la aplicación WinEpi Tasas), desde el nacimiento al destete en el estado Táchira, fueron en total de $29,29 \%$. Con un valor para la zona norte de $17,8 \%$ y para la zona sur de $42,14 \%$.

Valoración de la exposición a la infección por Cryptosporidium spp.como factor de riesgo para la presentación de diarreas en terneros del estado Táchira en Venezuela

El odds ratio (OR), la razón de prevalencia (RP), el riesgo atribuible de la población (RA), la fracción etiológica (FE) y la fracción atribuible de la población (FAP), en referencia al riesgo de presentación de diarreas de acuerdo con la exposición de los terneros al Cryptosporidium spp., se muestran en el Cuadro 1.

Cuadro 1. Comprobación por aproximación logarítmica y de $\chi^{2}$ para el odds ratio y razón de prevalencia para el factor exposición a Cryptosporidium spp. con relación a la diarrea en terneros del Estado Táchira, Venezuela. 2008.

Table 1. Logarithmic approximation and $\chi^{2}$ for odds ratio and prevalence ratio related for the exposure factor to Cryptosporidium spp. in relation with diarrhea in calves in the Tachira state, Venezuela. 2008.

\begin{tabular}{cccccc}
\hline & Aproximación & logarítmica & Aproximación & $\chi^{2}$ \\
\hline Límites & inf. & sup. & inf. & sup. \\
\hline OR & 2,275 & 1,399 & 3,700 & 1,406 & 3,68 \\
RP & 1,683 & 1,253 & 2,259 & 1,240 & 2,28 \\
\hline RA & $0,188(18,8 \%)$ & & & \\
FE & $0,406(40,6 \%)$ & & & \\
FAP & $0,167(16,7 \%)$ & & & \\
\hline
\end{tabular}

OR: razón de momios; RP: razón de prevalencias; RA: riesgo atribuible de la población; FE: fracción etiológica; FAP: fracción atribuible de la población / OR: odds ratio; RP: prevalence ratio; RA: attributable population risk; FE: etiological fraction; FAP: attributable population fraction.

Calculado con la aplicación Win Episcope/ Calculated by Win Episcope application.

Existe un grado de asociación entre la presencia del Cryptosporidium spp. y la presentación de diarreas en terneros del estado Táchira (Cuadro 1). Tanto el OR como la RP, señalan valores superiores a la unidad en forma estadísticamente significativa según las aproximaciones logarítmicas y de chi cuadrado $\left(\chi^{2}\right)$. Esto sugiere que Cryptosporidium spp. representa un factor de riesgo para la manifestación clínica de diarreas en el estado Táchira, por lo que, puede guardar relación con los signos presentados en un porcentaje importante de los animales muestreados. Se sugiere, además, que en el estado Táchira los terneros de corta edad expuestos a Cryptosporidium 
spp. presentaron un $18,8 \%$ de riesgo adicional de presentar diarrea, como lo señala el RA que representa la expresión de la relación de la exposición al parásito con la respuesta clínica.

Valoración de la edad en meses como factor de riesgo para la presentación de la infección por Cryptosporidium spp. en terneros

Al discriminar por edades las frecuencias de individuos positivos, se nota claramente un mayor número de terneros parasitados en los grupos de menor edad (Figura 1); y al tomar en cuenta la positividad a Cryptosporidium como variable de respuesta frente al factor edad en meses, considerado como un factor de riesgo, se estableció el estudio de regresión logística presentado en el Cuadro 2.

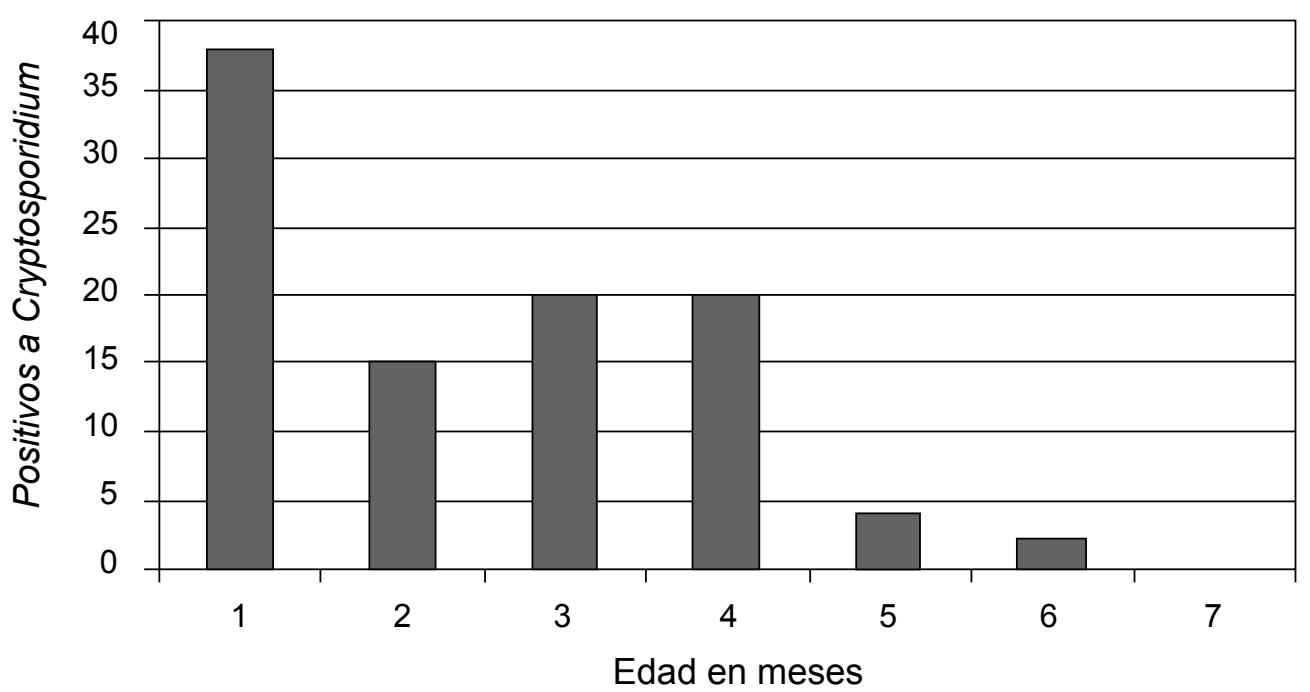

Figura 1. Distribución de las frecuencias de terneros positivos a Cryptosporidium spp. discriminados por edad en meses. Estado Táchira, Venezuela. 2008.

Figure 1. Distribution of the frequency of positivity calves to Cryptosporidium spp. discriminate by age in months. Tachira state, Venezuela. 2008.

Cuadro 2. Estudio de regresión logística relacionando la positividad a Cryptosporidium spp. en función de la edad de los terneros en el estado Táchira, Venezuela. 2008.

Table 2. Logistic regression study relating positivity to Cryptosporidium spp. depending on the age of the calves in the Tachira state, Venezuela. 2008.

\begin{tabular}{cccccccc}
\hline Positividad / función & OR & $\mathbf{9 5 \%}$ & I.C. & Coef. & S. E. & z & p \\
\hline Grupo edad & 0,69 & 0,59 & 0,81 & $-0,36$ & 0,082 & $-4,42$ & 0,000 \\
Constante & $*$ & $*$ & $*$ & 0,16 & 0,250 & 0,64 & 0,522 \\
\hline
\end{tabular}

OR: razón de momios; 95\%: límite superior; I.C.: intervalo de confianza; Coef.: coeficiente; S.E.: error estándar; z: valor de z; p: valor de p / OR: odds ratio; 95\%: upper limit; I.C.: confidence interval; Coef.: coefficient; S.E.: standard error; z: z value; p: p value. 
El análisis sugiere que, a mayor edad, en igualdad de condiciones ambientales, hay menos probabilidades de que las muestras resulten positivas al examen coprológico, de manera que el incremento de la edad en meses se comportó como un factor protector para la presencia de Cryptosporidium spp. en las muestras de heces. Se observa una relación altamente significativa $(\mathrm{OR}<1 ; \mathrm{p}<0,01)$ entre la edad y la excreción del parásito en las heces (Cuadro 2). Los terneros más jóvenes presentaron mayores probabilidades de resultar positivos al diagnóstico de este protozoario (Figura 1), coincidiendo con la característica autolimitante de esta infección.

Análisis multifactorial de la exposición a la infección por Cryptosporidium spp. Interacción con la edad, sexo y zona, como factores de riesgo para la presentación de diarreas en terneros del estado Táchira en Venezuela

Al analizar la relación entre la positividad a Cryptosporidium spp. y la presentación de diarreas, esta vez en forma multifactorial, considerando factores como la edad, zona geográfica y sexo se obtuvieron los resultados presentados en el Cuadro 3. Además, se calcularon interacciones entre positividad $\times$ edad, positividad $\times$ sexo y positividad $\times$ zona.

Cuadro 3. Análisis de regresión logística multivariada para el efecto de la positividad a Cryptosporidium spp. Edad en meses, sexo y zona, para la presentación de diarrea como variable resultado en terneros del Estado Táchira, Venezuela. 2008.

Table 3. Multivariate logistic regression analysis for the effect of positivity to Cryptosporidium spp. Age in months, gender, and area for the presentation of diarrhea as an outcome variable in calves in the Tachira state from Venezuela. 2008.

\begin{tabular}{cccccccc}
\hline Diarrea /factores & OR & $\mathbf{9 5 \%}$ & I.C. & Coef. & S. E. & z & p \\
\hline Positividad (+) & 8,29 & 1,14 & 60,36 & 2,11 & 1,01 & 2,09 & 0,036 \\
Grupo edad & 0,50 & 0,39 & 0,64 & $-0,70$ & 0,13 & 5,57 & 0,000 \\
Sexo (M/H) & 0,47 & 0,25 & 0,89 & $-0,76$ & 0,33 & 2,30 & 0,021 \\
Zona (N/S) & 1,16 & 0,62 & 2,20 & 0,15 & 0,32 & 0,47 & 0,638 \\
$(+) \times$ edad & 0,74 & 0,46 & 1,20 & $-0,30$ & 0,25 & 1,22 & 0,222 \\
$(+) \times$ sexo & 2,51 & 0,72 & 8,79 & 0,92 & 0,64 & 1,44 & 0,149 \\
$(+) \times$ zona & 0,13 & 0,03 & 0,57 & $-2,02$ & 0,74 & 2,72 & 0,007 \\
Constante & - & - & - & 1,34 & 0,44 & 3,05 & 0,002 \\
\hline
\end{tabular}

OR: razón de momios; 95\%: límite superior; I.C.: Intervalo de confianza; Coef.: coeficiente; S.E.: error estándar; z: valor de z; p: valor de p / OR: odds ratio; 95\%: upper limit; I.C.: confidence interval; Coef.: coefficient; S.E.: standard error; z: $z$ value; p: p value.

Calculado con Epi-info ${ }^{\mathrm{TM}} /$ Calculated by Epi-info ${ }^{\mathrm{TM}}$.

Con respecto a la presentación de diarreas, de los cálculos reflejados en el Cuadro 3, se sugiere que la exposición expresada como positividad a Cryptosporidium spp. es un factor de riesgo con 8,29 (OR>1) veces de exceso de ventaja para la presentación de signos de diarrea estando expuestos al parásito $(\mathrm{p}<0,05)$.

La edad en meses se comporta en el análisis multifactorial nuevamente como un factor protector para la presentación de diarreas por cualquier causa (Figura 2), considerando que el OR $(0,498)$ fue estadística y significativamente inferior a la unidad $(\mathrm{p}<0,05)$.

Con respecto al sexo, los resultados presentados en el Cuadro 3 y Figura 3, sugieren que el macho podría tener menor riesgo a sufrir el síndrome diarreico por cualquier causa $(\mathrm{OR}<1 ; \mathrm{p}<0,05)$. Sin embargo, al estudiar la interacción entre las variables sexo $\times$ positividad, este riesgo desaparece, lo que indica que aún cuando las hembras 


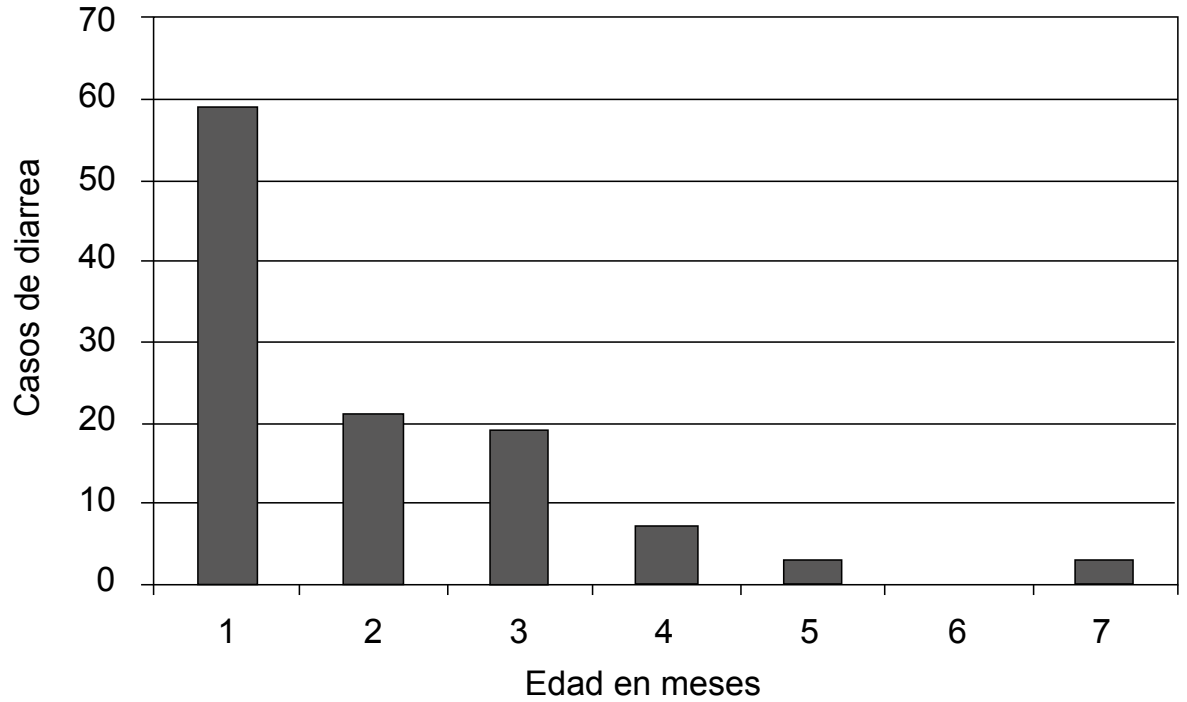

Figura 2. Distribución de la ocurrencia de diarreas por cualquier causa en terneros a diferentes edades. Estado Táchira, Venezuela. 2008.

Figure 2. Diarrhea occurrence distribution for any cause in calves of different ages. Tachira state, Venezuela. 2008.

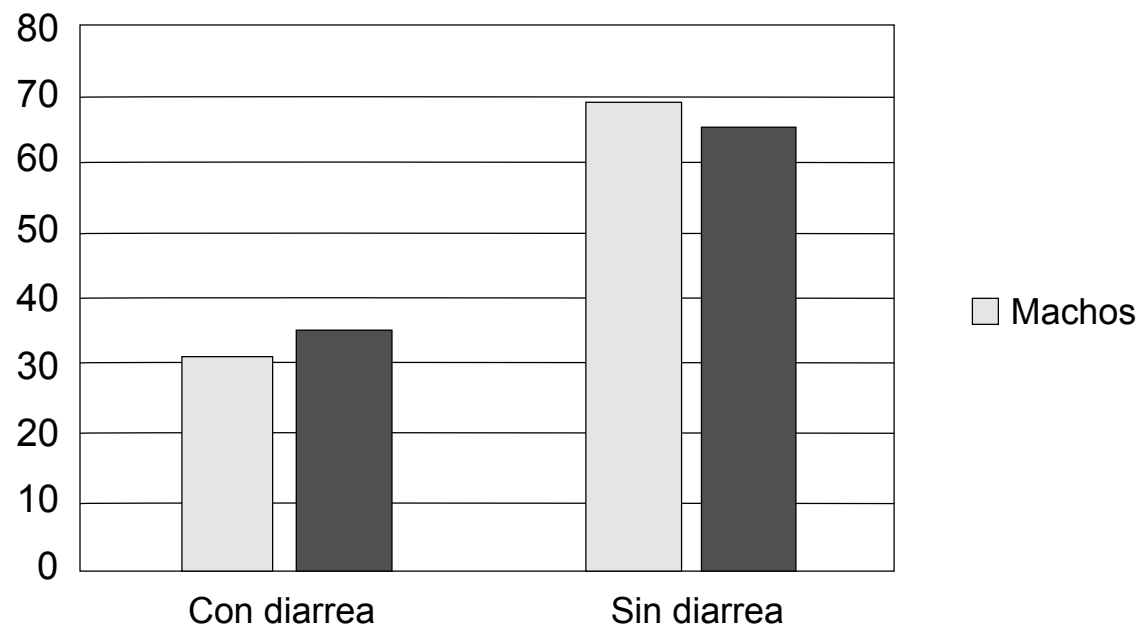

Figura 3. Presentación de diarreas por cualquier causa en terneros. Datos agrupados por sexo. Estado Táchira, Venezuela. 2008. Figure 3. Diarrhea occurrence for any cause in calves. Grouped data by gender. Tachira state, Venezuela. 2008.

ofrecían mayores posibilidades de sufrir diarrea por cualquier causa, esto se presentó independientemente de la presencia de Cryptosporidium en las heces ( $\mathrm{p}>0,05)$.

En cuanto a las zonas estudiadas, el 50,9\% de los casos de diarreas hallados correspondieron a la zona norte y el 49,1\% a la zona sur del estado Táchira, pero ninguna constituyó un factor de riesgo especial para la presentación de las diarreas por cualquier causa en terneros $(\mathrm{p}>0,05)$, aunque si se toma en cuenta la interacción entre las 
variables zona y positividad (zonaxpositividad, Cuadro 3), se sugiere que la ubicación en la zona norte, aún con una prevalencia menor que la zona sur, aumentó las probabilidades de que la presencia del Cryptosporidium en el animal pueda estar relacionada con la presentación de signos de diarrea. Se señala a la zona sur como de menores probabilidades de que se dé esta relación causal $(\mathrm{OR}<1 ; \mathrm{p}<0,05)$. En la zona norte, la sola presencia del Cryptosporidium spp. está relacionada significativamente con la presentación de diarreas como se observa en los resultados (Figura 4).

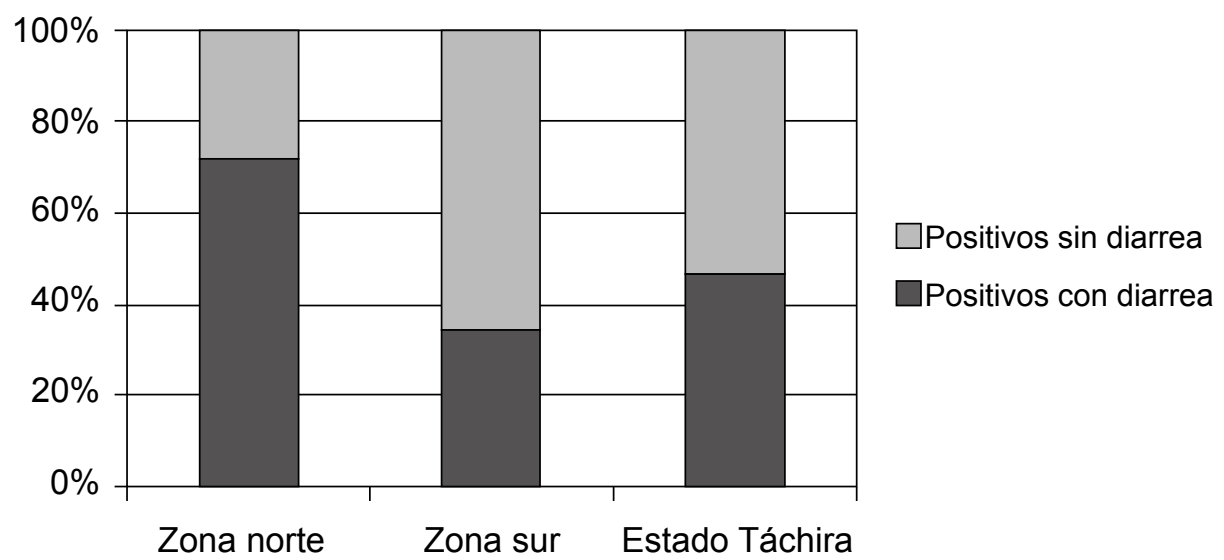

Figura 4. Positividad de terneros al Cryptosporidium spp. en relación con la presentación de diarreas en el Estado Táchira, zona norte y zona sur. Venezuela. 2008.

Figure 4. Positivity to Cryptosporidium spp. in calves related to diarrhea signs in the Tachira state, north and south zones. Venezuela. 2008.

\section{Discusión}

En el presente estudio, la tasa de infección para la zona sur $(43,8 \%)$ resultó más alta que para la zona norte $(17,8 \%)$, probablemente debido a las diferencias ambientales. Estos niveles de infección sugieren un riesgo de enfermedad en bovinos del estado Táchira y además, riesgo de enfermedad en seres humanos (Pérez-Schael et al., 1985; Hayes et al., 1989; Fayer, 1994; Wang et al., 2011; Maurya et al., 2013; Del-Coco et al., 2014), pudiendo representar un mayor riesgo zoonótico para la zona sur del estado.

Los niveles de infección para el estado Táchira obtenidos en el presente estudio $(29,3 \%)$, se pueden comparar con prevalencias reportadas en Venezuela por otras investigaciones: 57,5\% (Surumay y Pote, 1999); 29,3\% (Surumay y Alfaro, 2000); 22,1 (Surumay y Sandoval, 2000); 52,17\% (González y Moreno, 2003) y entre 26,06\% - 78,43\% (Alvarez el al, 2011). Probablemente las diferencias halladas entre autores sean principalmente debidas a las diferentes áreas geográficas y ecosistemas en donde se realizaron los estudios.

El 40,6\% de los terneros con diarrea, que además presentan criptosporidios en las muestras de heces, pueden guardar una relación causal en la que el parásito participe como responsable de estas manifestaciones clínicas, lo cual se expresa como la fracción etiológica (Wayne et al., 1997). El 16,7\% de la población total de terneros puede estar manifestando signos clínicos relacionados con la presencia de Cryptosporidium spp., porcentaje expresado como fracción atribuible de la población (Wayne et al., 1997; Llorca et al., 2001). 
Estos resultados coinciden con numerosos estudios que han señalado a este parásito como un agente importante en la producción de diarreas en terneros en Venezuela y otros países (Fayer y Ungar, 1986; Angus, 1987; Morgan, 1991; Díaz et al., 2004; Alvarez et al., 2011).

Los resultados coinciden con los de otros investigadores que señalan un mayor porcentaje de individuos infectados con C. parvum alrededor de las dos semanas de edad (Díaz et al., 2004; Alvarez et al., 2011). Igualmente coinciden en que, la criptosporidiosis bovina es relativa a la edad, siendo más común en edades menores de las tres semanas y más aún en los animales expuestos con edades entre los cuatro y quince días (Díaz et al, 2004).

Los terneros tienen menos riesgo de sufrir diarreas en la medida en que avanzan en edad, tal como lo señalan numerosos reportes de otros autores, en donde la edad de mayor riesgo para la presentación de diarreas indiferenciadas en terneros está comprendida desde el nacimiento hasta el primer mes de vida, pudiéndose combinar distintas causas para producir el síndrome (Morgan, 1991; Murphy y Andresen, 2000; Alvarez et al., 2011). No obstante, al analizar la interacción positividad $\times$ edad para la presentación o no de diarreas, se observó que no existieron diferencias significativas para el OR $(\mathrm{p}>0,05)$. Esto sugiere que estando en ese momento infectado el animal y excretando ooquistes por las heces, una mayor edad no disminuirá las probabilidades de que presente signos de diarrea por causa de este parásito. Aunque los autores consultados no señalan diferencias con respecto al sexo, las diferencias aquí presentadas podrían deberse a razones inmunitarias inherentes al sexo o a discriminación en el manejo y los cuidados ofrecidos a cada sexo en las explotaciones ganaderas (Vargas-Villalobos et al., 2014), en cuyo caso podrían estar interviniendo etiologías diferentes a Cryptosporidium spp.

En la zona sur, aunque se presentó una tasa más alta, pudieron estar presentes otros agentes etiológicos interviniendo con una relación causal estadísticamente más estrecha con los signos clínicos observados. También podría deberse a que como enfermedad autolimitante, en las zonas con mayor nivel de infección podrían darse mecanismos inmunitarios que tiendan a mantener la enfermedad en una forma subclínica en individuos infectados.

\section{Conclusiones}

La tasa de infección de Cryptosporidium spp. estimada en terneros del estado Táchira, es considerable tomando en cuenta el daño que puede causar en terneros en forma primaria o combinada con otros agentes etiológicos, además de las posibilidades de transmisión al ser humano, principalmente por contaminación de fuentes de agua de consumo o por condiciones ocupacionales.

La edad no fue determinante para que Cryptosporidium spp. estableciera una relación causal con las diarreas una vez que el ternero ha sido infectado, solo que la infección por este parásito fue más frecuente a edades más cercanas al nacimiento y principalmente durante el primer mes de vida.

La zona del estado Táchira no fue determinante para la presentación de diarreas por diferentes causas en los terneros, pero se encontró que en la zona norte hubo mayor probabilidad de que la diarrea se atribuyera a la infección por Cryptosporidium spp. entre las denominadas diarreas indiferenciadas en terneros de temprana edad.

En cuanto al sexo, aún cuando pareciera más susceptible la hembra a las diarreas, en general, esto resultó ser independiente de la presencia de Cryptosporidium spp. en las heces, por lo que, el estudio sugiere que el sexo no fue un factor importante para la presentación de criptosporidiosis.

\section{Literatura citada}

Aldeyarbi, H.M., N.M. Abu, and P. Karanis. 2016. Cryptosporidium and cryptosporidiosis: the African perspective. Environ. Sci. Pollut. Res. Int. 23:13811-13821. doi:10.1007/s11356-6746-6 
Alvarez, H.J., F. Mujica, y R. Meléndez. 2011. Prevalencia y viabilidad de ooquistes de Cryptosporidium spp. en becerras de fincas lecheras del estado Lara, Venezuela. Gaceta Cienc. Vet. 16:5-11.

Andrade, L., R. Fernández, G. González, y S. Rivera. 1986. Criptosporidiosis en la bolsa de Fabricio en pollos de engorde. En: Federación Nacional de Médicos Veterinarios, editor, Memorias de las IV Jornadas Nacionales Veterinarias. Federación Nacional de Médicos Veterinarios, Caracas, VEN. p. 105-106.

Angus, W. 1987. Criptosporidiosis in domestic animals and human. In Practice 9(2):47-50. doi:10.11336/inprect.9.2.47

Aranguren-Méndez, J., R. Román-Bravo, Y. Villasmil-Ontiveros, y F. Yánez. 2007. Evaluación genética de la ganadería mestiza doble propósito en Venezuela. Arch. Latinoam. Prod. Anim. 15:241-250.

Bhatia, B. 2000. Textbook of veterinary protozoology. Indian Council of Agricultural Research, New Delhi, IND.

Brar, A., P. Sood, N. Kaur, P. Singla, L., Sandhu, B. Gupta, and M. Chandra. 2017. Periurban outbreaks of bovine calf scours in Northern India caused by Cryptosporidium in association with other enteropathogens. Epid. Infect. 145:2717-2726. doi:10.1017/S0950268817001224

Castellanos, L., J. Pérez, y F. Urdaneta. 2013. Estructuras generales para registro contable de costos en sistemas de producción doble propósito en dos zonas del estado Táchira. Rev. Fac. Agron. (LUZ) 30:632-653.

Cho, Y., and K.J. Yoon. 2014. An overview of calf diarrhea-infectious etiology, diagnosis, and intervention. J. Vet. Sci.15:1-17. doi:10.4142/jvs.2014.15.1.1

Contreras, J.A. 2000. Enfermedades de los bovinos IV: diagnóstico tratamiento y control. Editorial Rapilit, Lara, VEN.

Del-Coco, V.F., M.A. Córdoba, G. Bilbao, A. Pinto, J.A. Basualdo, R. Fayer, and M. Santín. 2014. Cryptosporidium parvum GP60 subtypes in dairy cattle from Buenos Aires, Argentina. Res. Vet. Sci. 96:311-314. doi:10.1016/j.rvsc.2013.10.010

Díaz, A., L. Ramírez-Iglesia, O. Hernández, y N. Montilla. 2004. Cryptosporidium spp. en becerros neonatos de ganadería lechera y de doble propósito del estado Trujillo. Venezuela. Zoot. Trop. 22(2):125-132.

Fayer, R. 1994. Effect of high temperature on infectivity of Cryptosporidium parvum oocysts in water. Appl. Environ. Microbiol. 60:2732-2735.

Fayer, R. 2010. Taxonomy and species delimitation in Cryptosporidium. Exp. Parasitol. 124:90-97. doi:10.1016/j. expara.2009.03.005

Fayer, R., and B.L. Ungar. 1986. Cryptosporidium spp. and criptosporidiosis. Microbiol. Rev. 50:458-483.

González, L., y C. Moreno. 2003. Identificación de algunos patógenos causantes de diarrea en terneros en fincas de la Zona Norte del estado Táchira. Rev. Cient. UNET 15(2):1-8.

Hayes, E., T. Matte, and T. O’Brien. 1989. Large community outbreak of criptosporidiosis due to contamination of a filtered water supply. N. Engl. J. Med. 320:1372-1376. doi:10.1056/NEJM198905253202103

Leal, F., L. Avilán, y E. Valderrama. 1999. Áreas potenciales para el desarrollo del cacao en Venezuela. Rev. Agroalimen. 5(8):39-45.

Llorca, J., C. Fariñas-Álvarez, y M. Delgado-Rodríguez. 2001. Fracción atribuible poblacional: cálculo e interpretación. Gaceta Sanitaria 15:61-67.doi:10.1016/S0213-9111(01)71519-1

Mariné-Oliveira, G.F., M.C. do-Couto, M. de-Freitas-Lima, and T.C. do-Bomfim. 2016. Mussels (Perna perna) as bioindicator of environmental contamination by Cryptosporidium species with zoonotic potential. Int. J. Parasitol. Parasites Wildl. 5:28-33. doi:10.1016/j.ijppaw.2016.01.004 
Maurya, P.S., R. Garg, P.S. Banerjee, S. Kumar, R.L. Rakesh, K. Kundu, and O.K. Raina. 2013. Genotyping of Cryptosporidium species reveals prevalence of zoonotic $C$. parvum subtype in bovine calves of north India. Indian J. Anim. Sci. 83:10181023 .

Morgan, J. 1991. Epidemiology, diagnosis and control of undifferentiated calf diarrhoea. In Practice 12(1):17-20. doi:10.1136/ inpract.12.1.17

Murphy, G., und U. Andresen. 2000. Neuere aspekte zur beurteilung der eignung antidiarrhoischer Diätetika für das kalb. Der Praktische Tierartzt 81(3):246-257.

Orellana, J.J., J.S. Kaufman, and P. Pino. 2013. Interaction, synergy and antagonism in prospective epidemiological studies. Rev. Peru. Med. Exp. Salud Pública 30:687-690.

Pérez-Schael.J., Y. Boher, L. Mata, M. Pérez, and F.J. Tapia. 1985. Criptosporidiosis in Venezuelan children with acute diarrhea. J. Trop. Med. Hyg. 34:721-722. doi:10.4269/ajmh.1985.34.721

Rekha, K.M.H., G.C. Puttalakshmamma, and P.E. D'Souza. 2016. Comparison of different diagnostic techniques for the detection of cryptosporidiosis in bovines. Vet. World 9:211-215. doi:10.14202/vetworld.2016.211-215.

Ryan, U., R. Fayer, and L. Xiao. 2014. Cryptosporidium species in humans and animals: current understanding and research needs. Parasitology 141:1667-1685. doi:10.1017/S0031182014001085

SASA (Servicio Autónomo de Sanidad Agropecuaria). 2005. Población bovina según municipio y grupo etario segundo ciclo de vacunación antiaftosa año 2004. SASA, Estado Táchira, VEN.

Schiaffino, A., M. Rodríguez, M.I. Pasarin, E. Regidor, C. Borrell, and E. Fernández. 2003. Odds ratio or prevalence ratio? Their use in cross-sectional studies. Gaceta Sanitaria 17:70-74. doi:10.1016/S0213-9111(03)71694-X.

Silva-León, G.A. 2010. Tipos y subtipos climáticos de Venezuela. Universidad de Los Andes, Mérida, VEN.

Surumay, Q., y C. Alfaro. 2000. Cryptosporidium spp., en bovinos jóvenes de fincas de la región oriental de Venezuela. Rev. Invest. Clín. 41:245-250.

Surumay, Q., and L. Pote. 1999. Concurrent infections of Cryptosporidium and Giardia in dairy farms Mississippi State, USA. Rev. Cientif. 9:519-523.

Surumay, Q., y Y. Sandoval. 2000. Cryptosporidium spp. en bovinos jóvenes de fincas del estado Zulia, Venezuela. Vet. Trop. 25:73-80.

Vargas-Villalobos, O.A., J.A. Elizondo-Salazar, y L. Noguera-Solera. 2014. Factores relacionados con la falla en transferencia de inmunidad pasiva en terneras y terneros de lechería en la región central norte de Costa Rica. Nutr. Anim. Trop. 8:68-79.

Wang, R., H. Wang, Y. Sun, L. Zhang, F. Jian, M. Qi, C. Ning, and L. Xiao. 2011. Characteristics of cryptosporidium transmission in preweaned dairy cattle in Henan, China. J. Clin. Microbiol. 49:1077-1082. doi:10.1128/JCM02194-10

Wayne, S., A.H. Meek, y P. Willeberg. 1997. Epidemiología veterinaria: principios y métodos. Acribia, Zaragoza, ESP.

Wayne, S., A.H. Meek, y P. Willeberg. 1997. Epidemiología veterinaria: principios y métodos. Acribia, Zaragoza, ESP. 